\title{
Multisource Feedback (MSF): supporting professional development
}

\author{
Dr Julian Archer, NIHR ACL in Clinical Education, \\ Peninsula College of Medicine \&t Dentistry
}

The General Dental Council (GDC) is exploring the concept of revalidation. How then can dentists continue to improve their practice learning from identified personal goals, while providing evidence for a regulatory body and continuing to deliver a service? The medical profession has looked to Multisource Feedback (MSF) for much of its answer to this dilemma.

MSF, also known as $360^{\circ}$ feedback, can be defined as a process by which multiple individuals, representing discrete informant groups eg dental hygienists, provide feedback to recipients. The concept is based on the premise that practitioners' roles in multidisciplinary teams are fundamental and central to the provision of good client care. MSF assumes that practitioners can be individually assessed in a meaningful way across a number of competencies by this multidisciplinary team. Using advances in behavioural measurement theory, discriminating differences between practitioners can be quantified and reproduced. In addition, useful feedback can be provided as part of a quality improvement model to potentially improve client safety. Practically this involves the use of an online questionnaire distributed to the individual and their chosen raters. Feedback is then generated by collated comments and aggregating scores from the raters compared to self scores and ideally comparative cohort scores eg all practising dentists in the UK. This feedback should be supported by a trained facilitator.

\section{EMBRACED BY INDUSTRY AND MANAGEMENT}

MSF has a long history outside of healthcare starting in the military and education, developed in the psycho-occupational literatures throughout the 20th century and most significantly embraced by industry and management. Healthcare has informed the literature only over the last two decades.

MSF has been shown to be acceptable to practitioners and feasible being both short to complete and relatively unobtrusive for participants. It can identify poor performers and most importantly inform positive change in practice. However factors that influence any feedback response are complex and vary over time and context. Reactions of participants are influenced by perceptions of accuracy, credibility and usefulness of feedback. MSF systems therefore need quality assuring. In medicine MSF has been designed to assess a wide range of professional behaviours but consistently in the literature it appears that assessment by colleagues involves judgements about two overriding factors: clinical care and psychosocial behaviour; patients assess psychosocial behaviour only. Systematic raters leniency influenced further by occupation and seniority may undermine validity, as can the length of a working relationship and ethnicity of the participant. These factors amongst others need to be understood and quantified in order that the system is transparent, potentially modified and fairness assured. In addition, in order to make a judgement in relation to revalidation while also strengthening the validity for performance change, the use of any MSF system must demonstrate evidence of reliability. Modern psychometric techniques namely Generalisability theory help to estimate the numbers of assessments required to achieve a reliable result - namely that your recorded performance truly reflects your real performance. This does not remove subjectivity but helps to combine professional opinion in a meaningful way.

MSF is already being used to inform Foundation dental training programmes and forms part of the Diploma of Membership of the Joint Dental Faculties at The Royal College of Surgeons of England but little is known about its utility in general dental practice.

The National Clinical Assessment Service (NCAS) promotes patient safety by providing confidential advice and support to the NHS in situations where the performance of doctors and dentists is giving cause for concern. The employer/contracting body, or the practitioner themselves, can contact NCAS for help. Concerns raised can cover a range of issues about clinical capability, health and behaviour. The assessment takes primarily a developmental rather than a pass/fail approach, and seeks to identify areas of satisfactory practice as well as areas requiring improvement. As part of the assessment process NCAS utilise Multisource Feedback (MSF) and patient feedback assessment instruments. NCAS are looking to evaluate a new MSF instrument to enable dentists to gain a valuable insight into their own performance with anonymised feedback from their own multidisciplinary team. The feedback best supported by an appraiser can be used to inform CPD now while gaining valuable insight into MSF, should it be proposed for the revalidation of dentists in the future.

Conflicts of interest: Dr Julian Archer is the academic lead for clinical assessments at NCAS.

A list of references is available from the author at: julian. archer@pms.ac.uk

DOI: $10.1038 /$ sj.bdj.2008.1150 